\title{
Cluster Based Congestion Avoidance Strategy for Layered Wireless Sensor Network
}

\author{
Santhana Krishnan $\mathbf{B}^{1 *}$, Ramaswamy $\mathbf{M}^{1}$ \\ ${ }^{1}$ Department of Electrical Engineering, Annamalai University \\ Annamalai Nagar, Tamil Nadu, India. \\ * Corresponding author’s Email: ramyasugan@rediffmail.com
}

\begin{abstract}
The effort conjures to evolve a cluster based routing pattern with a view to address traffic congestion in a layered Wireless Sensor Network (WSN). The directive lays an emphasis to ensure the continuity of data flow between source and destination entities that reside in different layers of the network. It ridges to figure in the trends in data communication to foster an energy efficient traverse for the packets within the network. The formulation encompasses the use of a Cluster based approach to Ad-hoc On Demand Distance Vector (CAODV) routing pattern and pulls out a renewed platform for multipath data transfer. It attaches an extra significance in the choice of Cluster Head $(\mathrm{CH})$ in the sense that it requires to be a high energy node and serves as a pivotal focus for rerouting the data on the occurrence of an obstructionist environment. The performance examined using Network Simulator (NS2) exhibits admirable levels for the indices to claim the suitability of the scheme for use in real world applications.
\end{abstract}

Keywords: CAODV; Congestion avoidance; Energy efficient; Layered network; Performance indices; NS2.

\section{Introduction}

A wireless communication transceiver forms part of a sensor device along with a reasonably powerful processor to conceive applications where hundreds of inexpensive sensor nodes can be deployed and constitute a self organizing Wireless Sensor Network (WSN) [1]. Rapid technological advances in wireless communication endow it to be suitable for low cost and low complexity miniaturized sensor devices to capture environmental and tactical data for transmitting them back to a Base Station (BS) [2].The recent years continue to witness an upsurge in the use of WSN in applications that include environmental monitoring and military field surveillance.

The sensors in different regions of the field can collaborate to aggregate their data and provide more accurate reports about their local regions. In addition to improving the fidelity of the reported measurements, the data aggregation reduces the communication overhead in the network, leading to significant energy savings. However the dense deployment and unattended nature of WSNs make it quite difficult to recharge node batteries and castigate energy efficiency as a major design goal in these networks [3].

The fundamental activity spreads to forge a communication pattern through which data transfer can take place among the sensor nodes. The theory of routing refers to propound mechanisms for effective migration of data in a state that includes node mobility and node failures. The hierarchical routing is an efficient way to lower energy consumption by performing data aggregation and therefore decreases the number of transmitted messages [4].

The WSN forges to group nodes of the same hop count to the sink in different layers to create a layered architecture. The arrangement of the nodes along with the location of the sink decides the number of layers and the number of nodes in each layer [5]. Most of the existing studies consider the resource allocation problem at each layer separately with a priori information about the conditions of other layers. Due to the fact that a node's power supply is commonly a battery, enormous efforts 
appear to fo- cus on energy aware protocols in order to maximize the lifetime of the WSN.

Congestion arises in the event of the offered load exceeding the available capacity of the network. It coerces to increase the burst nature of data traffic resulting in large number of packet drops due to buffer overflow in the network layer and an increase in packet delivery latency [6] and [7]. It encounters as a result a fall in the overall network throughput together with an energy waste in the highly energy constrained WSN [8].

A multi-layer architecture with nodes at each layer interconnected as a graph has been framed for large WSN [9]. A simple fault tolerant and decentralized routing theory has been traced to offer easy extendibility. Agent based peer to peer layered system architecture has been developed for the transfer of data in WSNs [10]. The peers in the application layer, database layer and network layer have been designed to exchange data request, query execution plan and routing information respectively. A cloud particle swarm optimization algorithm has been combined with clustering technique to optimize the performance of WSN [11]. A search process suitable for double layer network has been laid to balance the energy consumption and prolong the network life cycle. Energy efficient distributed dynamics diffusion routing scheme has been introduced to realize the upper bound on performance in WSN [12]. The simulation results have been compared with its counterpart in terms of system life time, power dissipation and simplicity. A hierarchical strategy has been detailed to provide different channel access opportunity to different nodes based on the distribution of traffic loads [13]. The results have been related to reduce packet loss ratio, improve throughput and gained energy saving in WSN.

A load balancing algorithm has been proposed for a WSN to increase the network life time and reduce the delay in the delivery of packets [14]. The e- nergy aware approach has been tailored to construct the lower chain in each cluster and avoid the formation of long link between neighboring nodes. A distributed congestion avoidance method has been proposed to suite application specific nature of WSN [15]. The methodology has been formulated to use the ratio of the number of downstream and upstream node along with available queue cycles of the downstream node to detect incipient congestion. The detailed design, implementation and evaluation of congestion detection and avoidance mechanism (CODA) have been presented to enhance the energy efficiency of WSN
[16]. The performance benefits and practical engineering challenges of CODA have been experimented in sensor network test bed using Carrier Sense Multiple Accesses (CSMA).

The WSNs often experience severe network congestion due to the limited buffer capacity of the sensor nodes and limited capacity of the shared wireless medium [17]. It espouses an exquisite need to articulate methods of transferring data in the purview of a layered network.

\section{Problem Description}

The primary theory echoes to formulate a mechanism bestowed to ensure an uninterrupted transfer of data from source to destination nodes in a layered WSN. The methodology entails the incorporation of a Cluster based philosophy to an Ad-hoc On Demand Distance Vector routing pattern (CAODV) to incite an energy efficient approach and enhance the lifetime of the network [18]. It orients to route the packets through a high energy Cluster Head $(\mathrm{CH})$ node along with a facility to re-route them on the occurrence of congestion in the layered network [19]. The scheme investigates the performance metrics using Network Simulator (NS2) to establish its merits over other routing patterns and forge to bring about a new perspective in this mode of communication.

\section{Proposed Approach}

The emphasis revolves around the formation of a procedure that facilitates to address traffic congestion that may arise either due to an increase in the packet size beyond the capacity of the $\mathrm{CH}$ to handle or a disruption in the path between three source and destination entities located in two separate layers. In spite of the fact that hierarchical data transfer strategies do claim significant savings in total energy consumption, the creation of cluster based approaches promises to enhance system scalability and network lifetime. The sensor nodes close to each other group themselves into a cluster and the nodes in the same layer select a node in its adjacent layer closer to the sink as the forwarding node called $\mathrm{CH}$. The $\mathrm{CH}$ in the layered architecture locates itself closer to the sink and relays the data to the sink with lower energy consumption.

A node associates with a $\mathrm{CH}$ that is available in the presence of multiple heads in its communication vicinity and accepts the association of a member node only if its inclusion does not exceed its capacity constraint. The load therefore forages to be a secondary 
metric and perpetuates to distribute the traffic on each $\mathrm{CH}$, thus improvising the overall network usefulness. Each node lands at a head node in a single hop and in case if no head is available in a node's neighborhood, it may try to reach the nearest head by associating with a node that is already attached to a $\mathrm{CH}$.

The paradigm of clustering forays a two-tier hierarchy in which the $\mathrm{CHs}$ form the higher tier while member nodes form the lower tier. The CHs aggregate the data as a prelude to onward transmission and traverse them to the central base through other CHs. In light of the view that $\mathrm{CHs}$ often transmit data over longer distances, they become circumspect to lose more energy compared to the member nodes. The network may be reclustered periodically in order to select energyabundant nodes to serve as $\mathrm{CHs}$, thus distributing the load uniformly on all the nodes. Besides achieving energy efficiency, it fosters clustering to reduce channel contention and packet collisions, resulting in a higher throughput for the network.

The system considers randomly distributed nodes to move in a homogenous Poisson point process fashion over a rectangular field. The network is heterogeneous in the sense nodes of lower energy level E0 deployed with intensity N0 and that of higher energy level E1 deployed with an intensity N1 serve as normal nodes and CHs respectively. The nodes use Omni directional antenna and both function through single hop communication. The individual nodes send their raw measurement values to the CHs periodically which apply an appropriate aggregation function to produce a higher level message digest and subsequently send it to the BS.

The formulation uses the radio model [20], [21] shown in Fig.1, with Eelec as energy being dissipated to run the transmitter and or receiver circuitry and Eamp as the energy dissipation of the transmission amplifier.

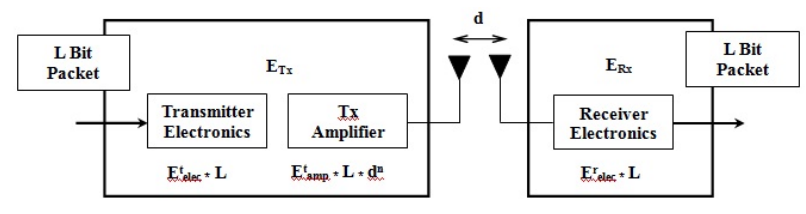

Figure.1 Radio Model

The transmission $E_{T x}$ and receiving $E_{R x}$ costs can be calculated using relations in Eq. (1) and Eq. (2).

$$
\begin{gathered}
E_{T x}(L, d)=L * E_{\text {telec }}+L * E_{\text {tamp }} * d n \\
E_{R x}(L, d)=L * E_{\text {relec }}
\end{gathered}
$$

where $L$ is the length of the transmitted/received message in bits, $d$ is the distance between transmitter and receiver node and $n$ is as the path loss exponent which is two for the free space model and can be up to six depending on the environment and network topology [22].

The CHs aggregate their cluster members data signals to produce a single representative signal, expending L-EDA for each L bit input signal, where EDA refers to the Energy for Data Aggregation. The optimum number of clusters Kopt for a cluster-based network with $\mathrm{N}$ sensor nodes distributed uniformly in an $M \times M$ region using the radio energy model in [21] can be calculated from Eq. (3).

$$
K_{o p t}=\frac{\sqrt{N}}{\sqrt{2 \pi}} \sqrt{\frac{\varepsilon_{f s}}{\varepsilon_{m p}}} \frac{M}{d_{t o B S}^{2}}
$$

where $d_{t o B S}$ denotes the distance from the cluster head node to the BS. The desired number of clusters can be obtained using expression (3) by substituting minimum and maximum values of dtoBS, the upper bound and the lower bounds. If the transmission distances fall below a threshold distance D0, it coerces to be a free space model where $\mathrm{n}$ takes the value of 2 and $\varepsilon_{f s}=$ Eamp. However for further dis-tances $\mathrm{n}$ increases to 4 and the model becomes multipath in nature with $\varepsilon_{m p}=$ Eamp.

The ratio between the amount of initial energy $E_{1}$ supplied to a $\mathrm{CH}$ and $P_{1}$, the energy spent by the head in each round seen in Eq.(4) decides the life time $T$.

$$
T=E_{1} / P_{1}
$$

Similarly if P1 is the amount of energy spent by a head node which includes the energy spent in receiving packets from all the normal nodes in it cluster, the energy spent in aggregating these packets into a single packet and transmitting it to the sink can be calculated from Eq. (5).

$$
P_{1}=E(N v) * E_{r 1}+E_{f}+E_{t 1}
$$

where $E(N v)$ is the expected number of nodes lying in each cluster, $E_{r 1}$ as seen in Eq. (6) relates to the energy spent in receiving packets from all the normal nodes in its cluster, $E_{f}$ is the energy spent in fusing the individual node's packets into a single aggregate and from Eq.(7). $\quad E_{t 1}$ is the energy spent in transmitting the aggregate value to the base station.

$$
E_{r 1}=E_{\text {relec }}\left(N_{0} / N_{1}\right) L
$$

where $L$ is the size of packets and $N_{0}$ is the normal node and $N_{1}$ is the Cluster Head Node. 
Similarly from Eq. (5), it is evident that,

$$
E_{t 1}=\left(E_{\text {relec }}+E_{\text {tamp }} * d n\right) L
$$

\section{Simulation Results}

The exercise endeavors to examine the performance of the network designed to transfer data between three sources and destination nodes located in two separate layers as seen in Figure 2. The scheme configures a network with two hundred intermediate mobile nodes distributed in a space of $1000 \mathrm{~m} \times 1000 \mathrm{~m}$ and the NS2 simulation study calculates the indices over a time frame of two hundred seconds using CAODV, Original AODV (OAODV), Improved AODV (IAODV) and Adaptive Cluster Based Routing Protocol (ACBRP).

The Figure 3 7 display the NS2 graphs for the different metrics that include the number of packets received, packet loss, throughput, energy expended and routing delay for a packet size of 3000. The number of packets appears to increase with time showcasing the highest increase for CAODV in Figure 3.

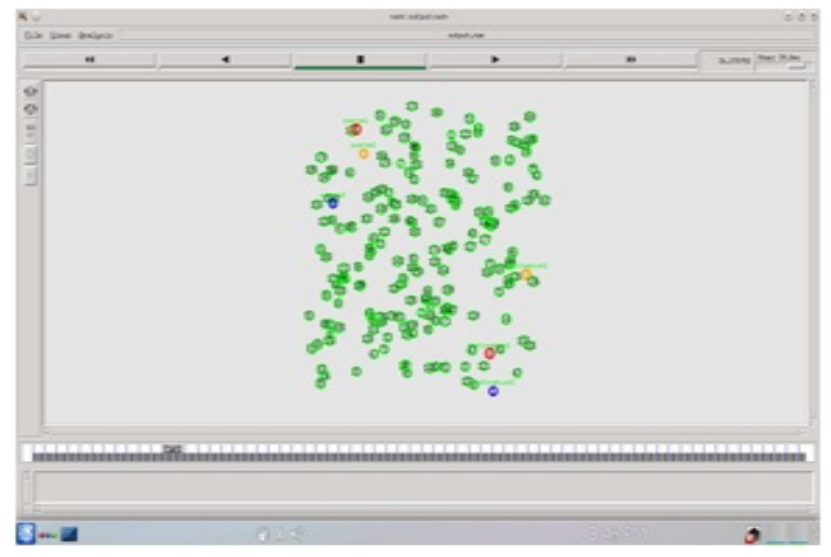

Figure 2 Network Model

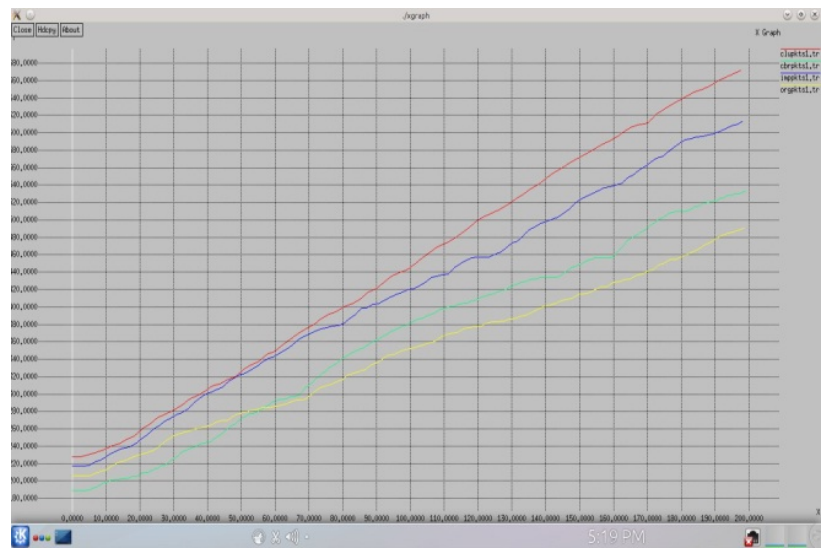

Figure 3 Packets Received vs Time
While the plot in Figure 4 depicts the minimum loss of packets for CAODV over other routing schemes, the one in Figure 5 exhibits the highest average throughput for CAODV to extradite its benefits on a similar platform.

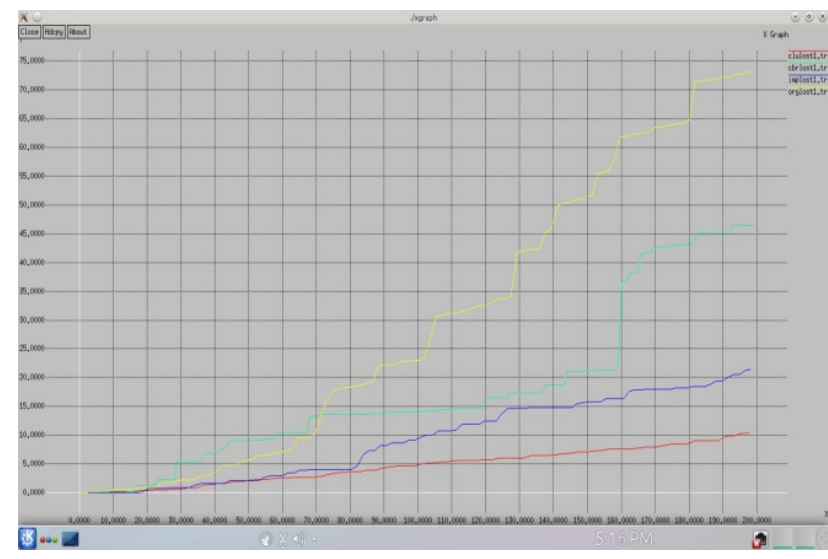

Figure 4 Packet Loss vs Time

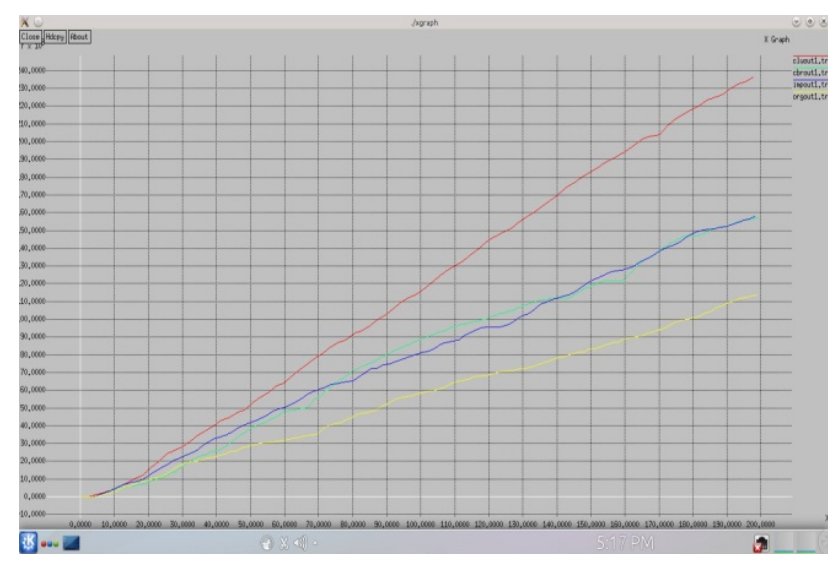

Figure 5 Throughput vs Time

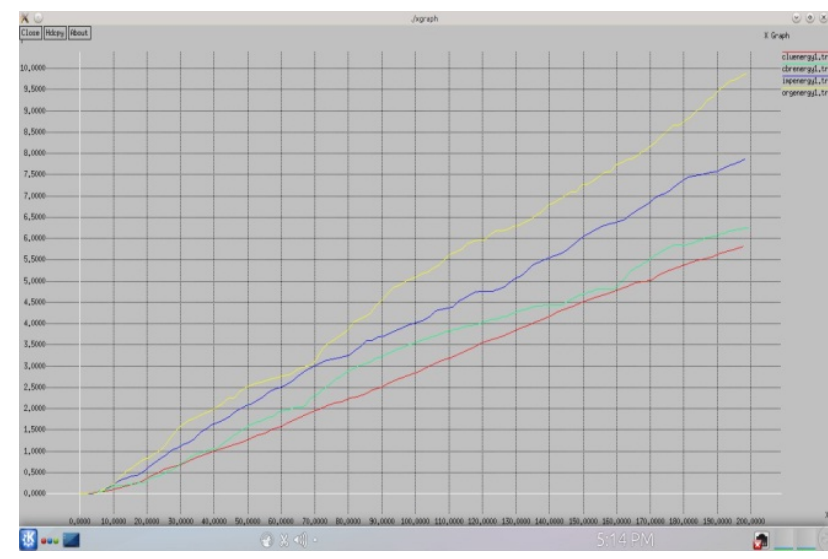

Figure 6 Energy Consumed vs Time

The energy pattern expressed in Figure 6 exhilarates the CAODV to etch out an energy efficient path for the packets in contrast with other routing techniques to claim an increase in the 
network lifetime. The graph in Figure 7 dwells with the minimum delay encountered with the flow of data for CAODV in comparison with the three other mechanisms.

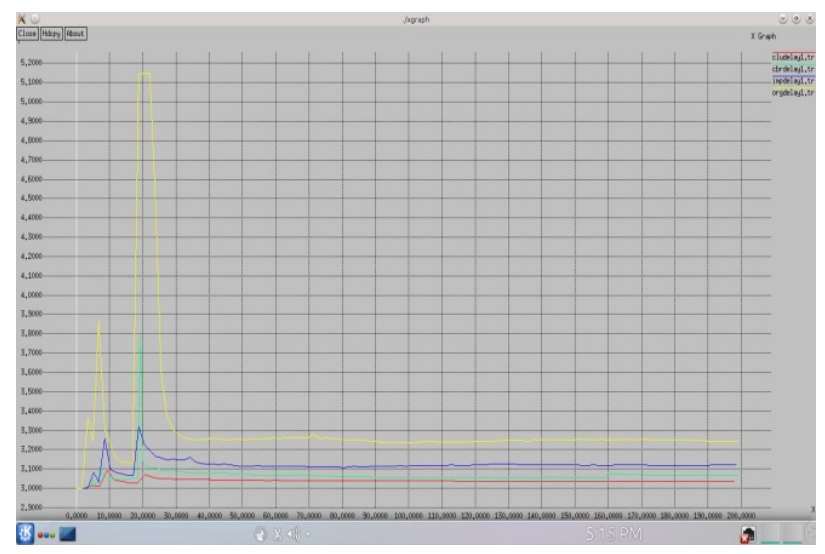

Figure 7 Routing Delay vs Time

The bar diagrams traced in Figure 8 through 14 foresee the suitability of the developed methodology to cater the large scale needs of the evolving communication media. The CAODV favors to transmit the highest number of packets accrue the lowest packet losses and enjoy the largest throughput for varying packet sizes ranging from 1000 to 5000 over the routing approaches as evinced from Figure 8 to 10.

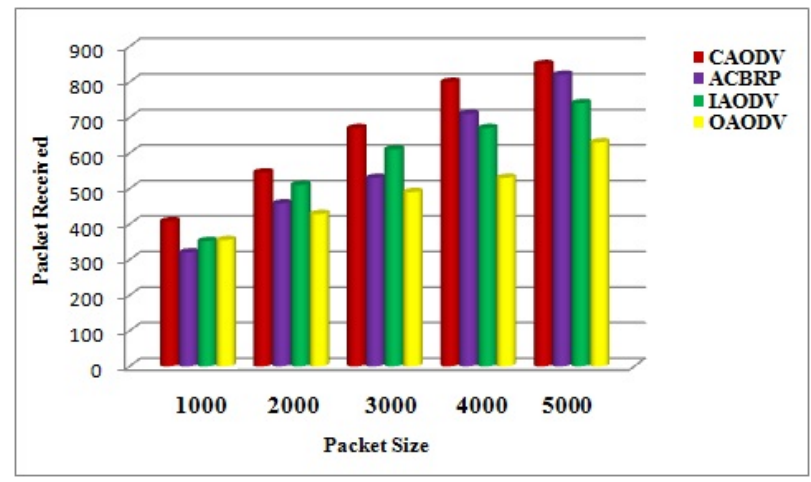

Figure 8 Packets Received vs Packet Sizes

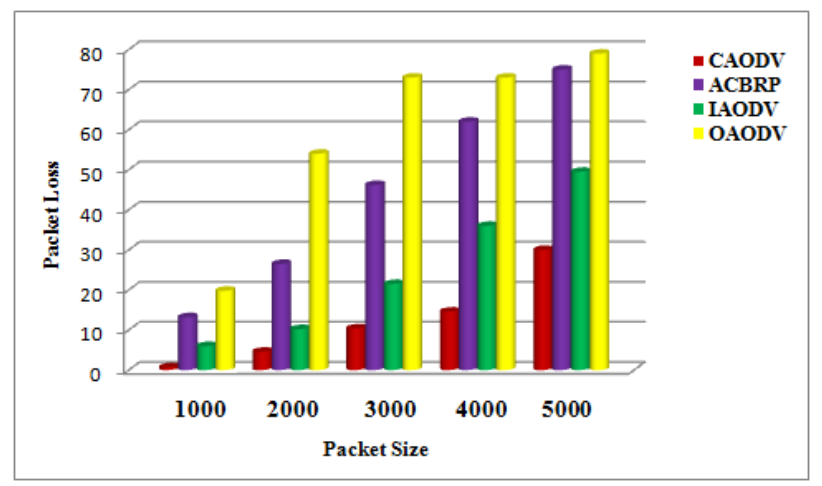

Figure 9 Packet Loss vs. Packet Sizes

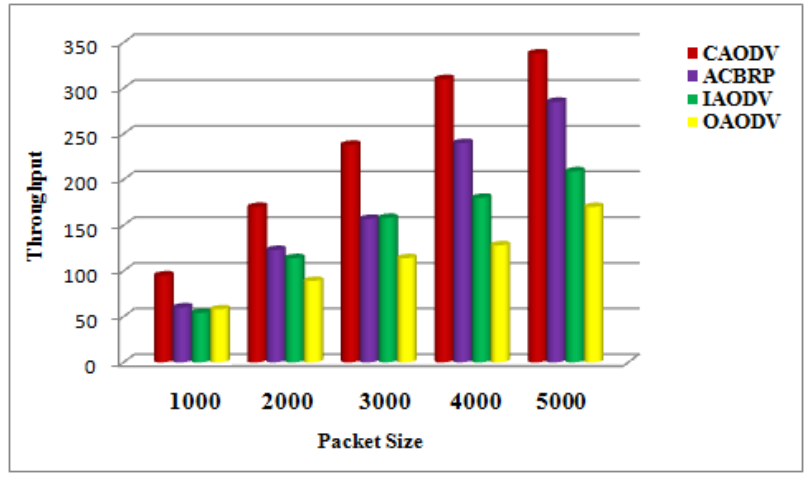

Figure 10 Throughput vs Packet Sizes

The benign fact relates to the bar chart in Figure 11 where the CAODV caste shows increasing energy expenditure for higher data sizes. However Figure 12 explains how the artifacts of CAODV encircle a lower delay for higher sized packet transfer in relation to other strategies. Figure 13 correlates to turn out the smallest Energy*Delay metric and allows raising the speed of transmission for CAODV over other routing patterns.

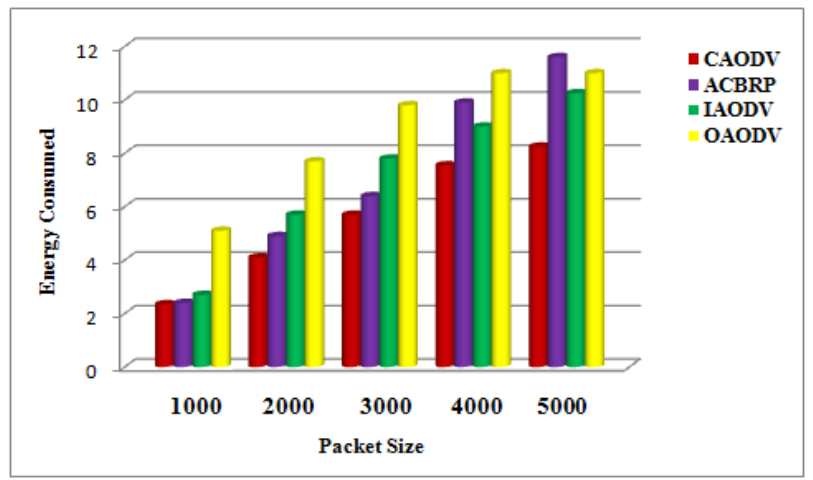

Figure 11 Energy Consumed vs Packet Sizes

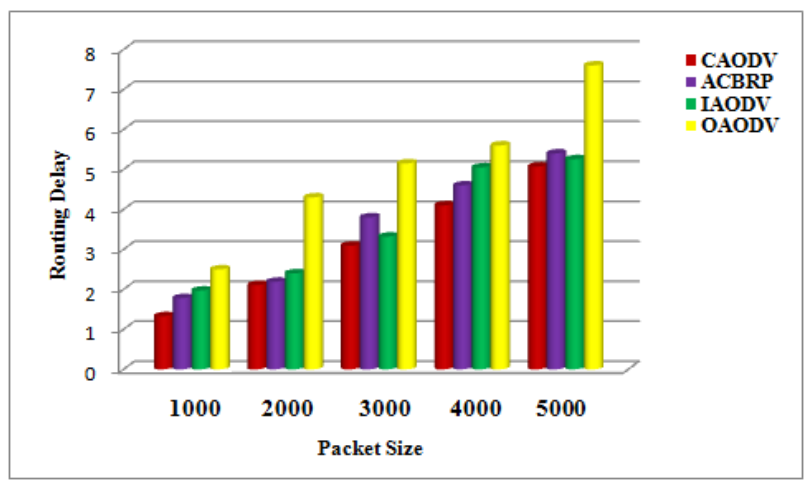

Figure 12 Routing Delay vs. Packet Sizes 


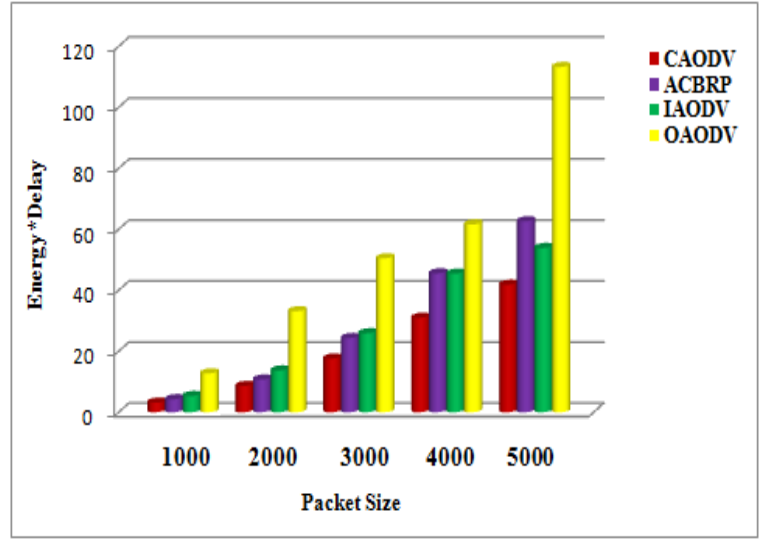

Figure 13 Energy*Delay vs. Packet Sizes

Though the line chart depicts the network Packet Delivery Ratio (PDR) in Figure14 to experience a slow fall for higher data sizes, still CAODV derives the largest PDR over other routing methods. The results endeavor to validate the fact that CAODV outperforms ACBRP and elucidates the benefits of clustered concepts in the process of routing.

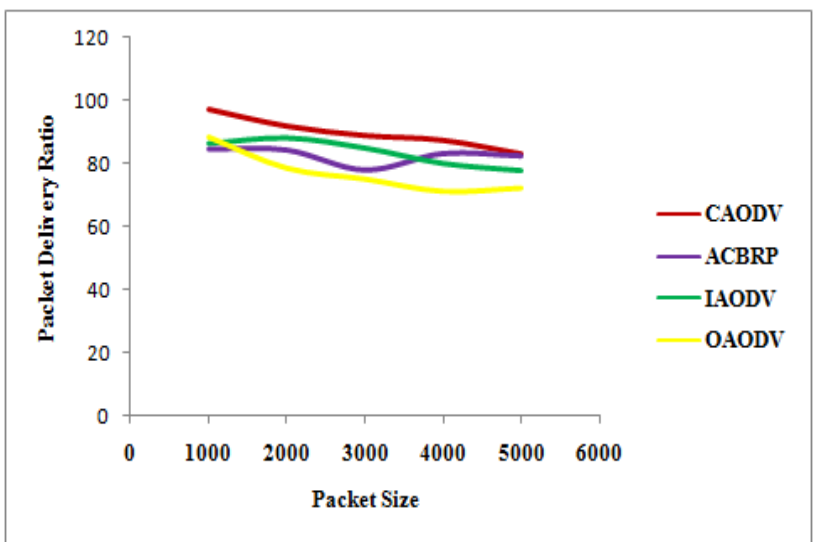

Figure 14 Packet Delivery Ratio vs Packet Sizes

The entries in Table.1 bring out the ability of the CAODV routing to arrive at the admirable indices for the network even in the event of occurrence of traffic congestion due to an increase in the buffer capacity of the $\mathrm{CH}$. Comparative study of the performance with and without traffic congestion reflects the benefits of rerouting the data through an enhancement in the performance.

\section{Conclusion}

A congestion avoidance strategy has been formulated to ensure a continuous flow of data in a layered WSN. The benefits of the proposition have been realized through a significant enhancement in the performance indices. The pivotal theme of a cluster has been intertwined with the principles of an Ad-hoc On Demand Vector routing to augur a different perspective in the management of traffic in the wireless domain. The elaborate study essayed on a NS2 platform has been focused to compare the performance of three other data transfer schemes to establish the merits of CAODV. The suitability of the proposed methodology to cater the needs of larger sized packets between three source and destination outfits that reside in different layers adds to its credibility. The simulation results have been portrayed to cleave out an energy efficient mechanism accrued using an increase in the number of packets received, throughput and a decrease in the delay and packet loss for CAODV. The consequent increase in life time of the layered network will go a long way in the use of CAODV. and facilitate to corner the realms of data communication.

\section{Acknowledgments}

The authors thank the authorities of Annamalai University for providing the necessary facilities in order to accomplish this piece of work.

Table 1 Performance Comparison

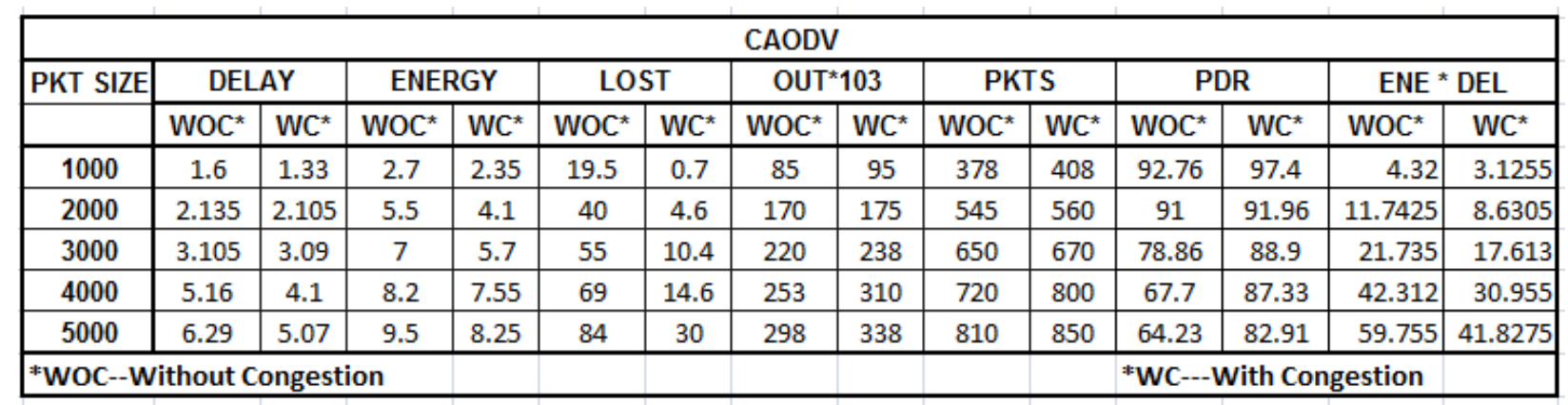




\section{References}

[1] G. J. Pottie and W. J. Kaiser, "Wireless integrated network sensors," Communications of the ACM, Vol. 43, No.5, pp.51-58, 2000.

[2] F. Akyildiz, W. Su, Y. Sankarasubramaniam, and E. Cayirci, "A survey on sensor networks”, IEEE Communications Magazine, Vol.40, pp.102-114, 2002.

[3] "Habitat Monitoring on Great Duck Island", in: http://www.greatduck island.net/, 2006.

[4] N. Amini, S. G. Miremadi and M. Fazeli, "A Hierarchical Routing Protocol for Energy Load Balancing in Wireless Sensor Networks”, IEEE Trans., pp. 1086- 1089, 2007.

[5] J. Ding, K. Sivalingam, R. Kashyapa, and L.J. Chuan, "A multi-layered architecture and protocols for large- scale wireless sensor networks”, in Proc. IEEE Semiannual Vehicular Technology Conf., pp. 1443-1447, 2003.

[6] B. Hull, K. Jamieson, and H. Balakrishnan, "Mitigating Congestion in Wireless Sensor Networks", in ACM SenSys, Baltimore, MD, pp.134-147, 2004.

[7] I. Demirkol, C. Ersoy, and F. Alagoz, "MAC Protocols for Wireless Sensor Networks: A Survey," in IEEE Communications Magazine, pp.115-121, 2006.

[8] C. Song, S. Hamid, and N. Guevara, "Improving Performance of MAC Layer by Using Congestion Control/Avoidance Methods in Wireless Network", in Proceedings of the 2001 ACM Symposiumon Applied Computing, pp.420-424, 2001.

[9] T. T. Huynh and C. S. Hong, “A Novel Multi-layer Architecture for Wireless Sensor Networks", in Korea Science \& Engineering Foundation, pp.11431146, 2003.

[10] E. Shakshuki, S. Hussain, A. W. Matin and A. R. Matin, "Agent-based peer-to-peer layered architectture for Data Transfer in Wireless Sensor Networks”, in IEEE Transaction, pp. 490-493, 2006.

[11] Z. Wu, Y. Nie, S. Qiang and H. Z. Wang, “ Double Layer Clustering Algorithm Based on CPSO for Wireless Sensor Networks”, in Information Technology Journal, Asian network for Scientific Information, pp.1737-1743, 2012.

[12] I. Raicu, L. Schwiebert and S. Fowler, "Local Load Balancing for Globally Efficient Routing in Wireless Sensor Networks”, in International Journal of Distributed Sensor Networks, Taylor \& Francis Inc., pp.163-185, 2005.

[13] Y. Liu, J. Pu, L. Shu and Z. Xiong, “A Hierarchical Congesstion Aviodance Alogorithm in Wireless Sensor Networks”, in International Journal of Inno- vative computing, information and Control, pp. 5199-5210, 2012.

[14] Y. Yu and G. Wei, "Energy Aware Routing Algorithm Based on Layered Chain in Wireless Sensor Network, ” IEEE Transaction, pp.2701-2704, 2007.

[15] M. Z. Ahmad and D. Turgut, "Congestion avoidance and fairness in wireless sensor networks", in IEEE GLOBECOM, proceedings, pp.1-6, 2008.

[16] C. Wan, S.B. Eisenman and A.T. Campbell, "CODA: Congestion Detection and Avoidance in Sensor Networks”, in SenSys'03, ACM, pp. 266-279, 2003.

[17] M. C. Vuran, V. C. Gungor, and O. B. Akan, “On the Interdependency of congestion and Contention in Wireless Sensor Networks", in ICST SenMetrics '05, 2005.

[18] B. S. Krishnan and M. Ramaswamy, "A New Cluster Based Protocol for Wireless Sensor Networks", in International Conference on Information Science and Application (ICISA-2011), IEEE Computer Society, pp. 1-8, 2011.

[19] B. S. Krishnan and M. Ramaswamy, "Cluster Based Data Transfer Strategy for Layered Wireless Sensor Network", in International journal of Computer Science and Issues, Vol.10, pp.184-189, 2013.

[20] W. R. Heinzelman, A. P. Chandrakasan, and H. Balakrishnan, "Energy efficient communication protocol for wireless microsensor networks," in Proc. of the 33rd IEEE International Conference on System Sciences, pp.1-10, 2000.

[21] W. B. Heinzelman, A. P. Chandrakasan, and H. Balakrishnan, "An application-specific protocol architecture for wireless microsensor networks", in IEEE Trans. on Wireless Communications, Vol.1, No.4, pp. 660-670, 2002.

[22] T. Rappaport, "Wireless Communications: Principles \& Practice”, Englewood Cliffs, NJ: Prentice-Hall, 1996. 